\title{
Apical Vertebral Column Resection with Sagittal Rotation and Controlled Anterior Opening and Posterior Closing Maneuver for the Treatment of Severe Post-Tubercular Kyphosis: Case Series and Literature Review
}

\author{
Sudhir Kumar Srivastava, Rishi Anil Aggarwal, Sunil Krishna Bhosale, \\ Kunal Roy, Pradip Sharad Nemade, Shaligram Purohit \\ Department of Orthopaedics, Seth GS Medical College and KEM Hospital, Mumbai, India
}

\begin{abstract}
Study Design: Retrospective case series.
Purpose: Describe the technique and evaluate the outcome of apical vertebral column resection (VCR) with sagittal rotation and anterior opening and posterior closing (AOPC) maneuver for correction of severe post-tubercular kyphosis (PTK).

Overview of Literature: The surgical procedures described for the correction of PTK are VCR, pedicle subtraction osteotomy, transpedicular decancellation osteotomy, and closing-opening osteotomy.

Methods: We retrospectively evaluated 21 patients who had been operated on with single stage apical VCR with AOPC maneuver. Radiographs were obtained before surgery and at regular follow-up intervals. These were used to calculate the angle of kyphosis. Back pain was rated using the Visual Analog Scale (VAS) and neurological status was graded using Frankel grading. Radiological outcome was assessed by the improvement in the angle of kyphosis and fusion following surgery. Neurological status was assessed using Frankel grading.

Results: The study included eight males and 13 females with a mean age of 21.9 and average follow-up time of 30.4 months. The average number of vertebral bodies destroyed was 2.57. Kyphosis was improved from a mean of $68.42^{\circ} \pm 13.23^{\circ}$ preoperative to $8.71^{\circ} \pm 4.58^{\circ}$ postoperative. The average correction achieved was $87.10 \%$. Preoperative VAS score improved from a mean of $6.38 \pm 0.92$ preoperative to $1.38 \pm 0.49$ postoperative. No patients had any sign of neurological deterioration. Seven out of eight patients with a preoperative neurological deficit improved following surgery. Two patients developed superficial wound maceration, one had persistent postoperative hypotension, and the other developed hemothorax. All patients recovered fully without a need for additional surgery.

Conclusions: Single stage simultaneous anterior column lengthening and posterior column shortening is an effective method for surgical correction of severe PTK.
\end{abstract}

Keywords: Vertebral column resection; Kyphosis; Post-tubercular; Osteotomy; Anterior opening posterior closing

Received Aug 29, 2018; Revised Oct 23, 2018; Accepted Oct 25, 2018

Corresponding author: Rishi Anil Aggarwal

Department of Orthopaedics, Seth GS Medical College and KEM Hospital, Parel, Mumbai-400012, Maharashtra, India

Tel: +91-9870775102, Fax: +91-22-2414-3435, E-mail: ris1987@gmail.com 


\section{Introduction}

Tuberculosis is a major healthcare problem in many developing countries, and tuberculosis of the spine (TBS) is one of the most common extra-pulmonary forms. More than two million people suffer from TBS worldwide [1], but with advancements in drug therapy, most cases can be dealt with ambulatory chemotherapy. Non-operatively treated patients have an average of $15^{\circ}$ increase in kyphosis and if left untreated, $3 \%-5 \%$ of these cases gradually progress to develop severe kyphosis [2]. Overlooked diagnoses, poor accessibility to healthcare, and socioeconomic factors are major reasons for the occurrence of such severe post-tubercular kyphosis (PTK).

PTK leads to significant physical and psychological morbidity. Persistent pain, costo-pelvic impingement, cardiopulmonary compromise, and late onset neurological deficits are problems associated with PTK. Poor cosmetic appearance associated with PTK is a major psychological burden due to a social stigma particularly in developing countries. Surgical correction of kyphosis will greatly improve these patients quality of life.

Untreated TBS, especially in children, leads to destruction of multiple vertebral bodies and subsequent shortening of the anterior column. Progressive kyphosis further adds to anterior column shortening and causes posterior column lengthening. Therefore, the goal of surgical procedures is to lengthen the anterior column, shorten the posterior column, or accomplish both. Surgical approaches available for treatment of kyphosis are: only anterior procedure [3] or only posterior procedures, like pedicle subtraction osteotomy (PSO) $[4,5]$, or combined posterioranterior procedures $[6,7]$. We describe our experience with single stage vertebral column resection (VCR) with sagittal rotation (SR) and controlled anterior opening and posterior closing (AOPC).

\section{Materials and Methods}

Between January 2007 and December 2014, we retrospectively reviewed the operating theatre reports, patient records, and radiographs of 21 consecutive patients who had surgery for PTK. We obtained approval from the institutional review committee of Orthopedics Seth GSMC and KEM Hospital (IRB approval no., 45/2015) before starting this study. Written and informed consent was given by patients for use of their clinical and radiological data for the purposes of this study.

Our indications for surgery were: presence of neurologic deficit $(n=8)$, progressive kyphosis $(n=9)$, intractable pain $(n=3)$, and cardiopulmonary distress $(n=1)$. We noted patient age, sex, diagnosis, levels involved, pre and postoperative angle of kyphosis ( $\mathrm{K}$ angle), loss of correction at follow-up, pre- and postoperative neurological status (Frankel grading), pre- and postoperative pain assessed by Visual Analog Scale (VAS) score, estimated blood loss, and postoperative complications.

\section{Radiological evaluation}

Preoperative radiographs included a standing anteroposterior (AP) view and lateral view of the dorso-lumbar spine. Preoperative computed tomography (CT) scan and magnetic resonance imaging were performed in all patients. Bone density scan by dual-energy X-ray absorptiometry (DEXA) was performed in all patients prior to surgery. An AP and lateral view radiograph of the dorsolumbar spine was taken postoperatively and during follow-up at 3, 6, 12, and 24 months. Postoperative radiographs were taken in the supine position and subsequent radiographs at followup were taken in the standing position. The $\mathrm{K}$ angle was calculated by drawing lines from upper border of first normal vertebra above and lower border of first normal vertebra below the diseased area (Fig. 1A). A CT scan was performed at 1 year postoperatively. Anterior fusion was assessed by presence of bridging bone between the verte-
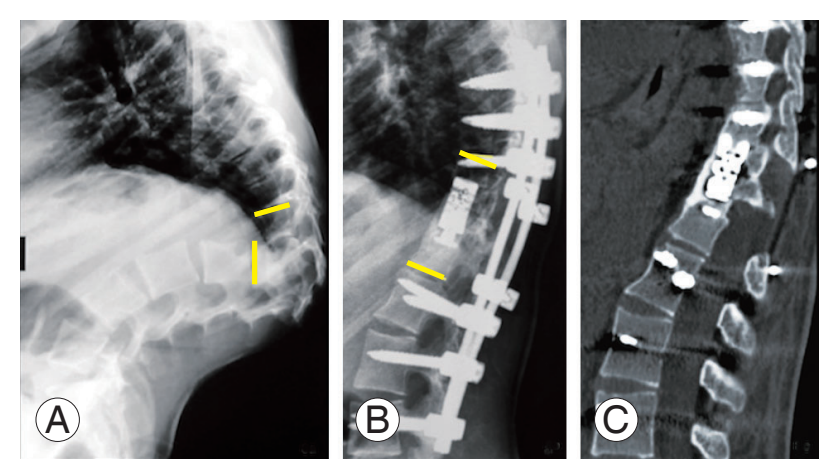

Fig. 1. (A) Preoperative radiograph showing severe kyphosis. Angle of kyphosis is calculated by drawing lines from the upper border of the first normal vertebra (upper solid line) above and the lower border of the first normal vertebra below the diseased area (lower solid line). (B, C) Postoperative radiograph showing correction of the severe kyphosis. Posterior instrumentation has been done with pedicular screws and anterior reconstruction with cage and morselized bone graft. 
bra immediately proximal and distal to the reconstructed area. The first author, who is also the primary surgeon of all the cases, performed the assessment of fusion on all radiographs. Calculation of $\mathrm{K}$ angle was performed by a radiologist who is not a part of the study.

\section{Surgical procedure}

Following induction of general anesthesia, the patient was placed in the prone position. Four bolsters were used, two were placed proximal and two distal to the break in the table. When necessary, the table may be appropriately broken to accommodate the deformity. The break in table also assists in extension of proximal segment and aids in the correction of the deformity.

Aside from all routine investigations, patients were preoperatively subjected to the following procedures for risk stratification and optimization: (1) pulmonary function test, arterial blood gas (ABG) analysis, chest consultation; (2) two-dimensional echocardiography, electrocardiogram (ECG), and cardiology consultation; (3) fundoscopy and intraocular pressure measurement; (4) counseling by psychiatrist and dietician; (5) neurology consult; (6) Stagnara wake-up test, explained in detail during the preoperative assessment, on the day prior to and on the morning of surgery.

All patients received iron therapy and a high protein diet from the time they first visited the outpatient department.

All cases were performed under general anesthesia. All patients were monitored for ECG, oxygen saturation, endtidal $\mathrm{CO}_{2}$, invasive blood pressure, core temperature, $\mathrm{ABG}$ and serum electrolytes were monitored intraoperatively in all patients. Serum calcium, prothrombin time, and international normalized ratio (INR) were monitored when the blood loss exceeded $30 \%$ of blood volume. The antifibrinolytic agent used was tranexamic acid with $10 \mathrm{~mL} / \mathrm{kg}$ bolus followed by a continuous infusion $1 \mathrm{mg} / \mathrm{kg} / \mathrm{hr}$ until skin closure. Propofol, dexmedetomidine, and fentanyl were routinely used as intravenous drugs. Sevoflurane or desflurane were used as inhalational agents. Rocuronium, vecuronium, and atracurium were used as muscle relaxants. Mean arterial pressure was always kept above $60 \mathrm{~mm}$ $\mathrm{Hg}$ and circulating hemoglobin was kept approximately at $8 \mathrm{gm} \%$. Short acting drugs such as propofol, atracurium, and desflurane were the choices before the wake-up test. Pregabalin, Paracetamol, fentanyl, tramadol, and ketoro- lac were used in the perioperative period for analgesia.

To reduce intraoperative bleeding, acute normovolemic hemodilution was practiced with tetrastarch or gelatin 20 $\mathrm{mL} / \mathrm{kg}$ at induction. Blood products were used at the earliest. Fresh frozen plasma (FFP) was used when the INR $>1.5$, and packed cell volume, FFP, and platelet in a 1:1:1 was used when there was a massive transfusion or when the surgeon felt that the bleeding was rapid.

\section{Fixation and decompression (column resection)}

The midline incision was made, and the standard posterior exposure was done. Adequate lateral exposure beyond the transverse processes was done at the site of deformity to facilitate exposure of the ribs. Under fluoroscopic guidance, pedicle screws were passed in a minimum of three levels proximal and distal to the deformity. Reduction screws were used in the pedicles proximal to the deformity. Following this, a temporary malleable aluminum rod was applied on one side.

The next step was to excise the bilateral ribs of vertebrae involved in internal gibbus. An incision was made on the periosteum of the rib with electro cautery. A periosteum elevator was then used to separate the rib from its periosteum. It was mandatory to stay strictly sub-periosteal to prevent injury to the parietal pleura. The rib was cut as lateral as possible and was excised along with its head. The same procedure was repeated on the opposite side. The pedicles and exiting nerve roots were then identified. In the dorsal spine, two or three nerve roots could be safely sacrificed by ligating them proximal to the dorsal root ganglion. Ligatures should be left long as they are used as landmarks and also to gently lift the dura during the terminal stages of resection of internal gibbus. The nerve roots in the lumbar spine cannot be ligated; hence, the procedure is challenging when attempted in the lumbar spine. We had one case where we performed the procedure in the lumbar spine, and the D12 nerve root was ligated. Far lateral decompression of L1 and L2 nerve roots was performed, and the roots were gently retracted to perform VCR.

After delineating the outer aspect of the pedicle, meticulous blunt dissection was continued on the surface of the vertebral body until all soft tissue was pushed anteriorly until the entire lateral and anterior surface of the vertebral body was free from the 2 oclock position to 10 o'clock. A catheter could then be passed from one side and removed from the other side to confirm complete clearance (Fig. 2). 


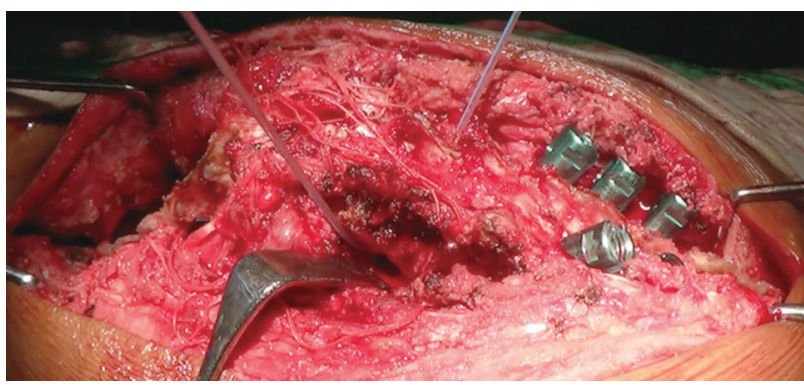

Fig. 2. Intraoperative image following the excision of the bilateral ribs of the vertebrae forming the internal gibbus. Following soft tissue dissection to free the anterior and lateral surface of the vertebra, a catheter is passed from one side and removed from the other side to ensure complete clearance of the soft tissues.

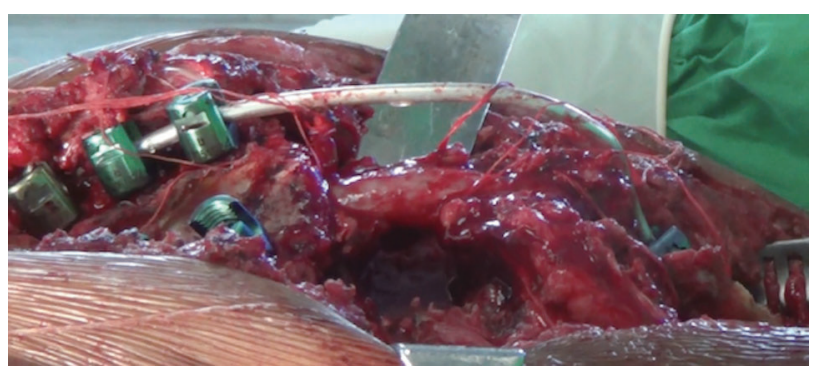

Fig. 3. Intraoperative image following vertebral column resection and bilateral costo-transversectomy. A temporary rod contoured to the shape of the deformity has been applied.

A mirror may also be used to confirm the same end result.

The transverse process, pedicles and anterior $2 / 3 \mathrm{rd}$ part of body of vertebrae forming the internal gibbus were then excised. The posterior cortex of vertebrae forming internal gibbus was not removed at this stage. Following this, laminectomy was performed on all vertebrae involved in the internal gibbus. Part of the lamina of the vertebra immediately proximal and distal to internal gibbus was also excised to make room for the spinal cord. A temporary rod was then applied on the other side prior to completing the VCR.

Then, VCR was completed by removal of posterior cortex. The cortex was thinned out using a burr or curette. Once only a thin shell of posterior cortex was remaining, it was pushed gently anteriorly using a blunt periosteum or penfield and then excised. Following VCR, the spinal cord was seen hanging freely between the proximal and distal segments (Fig. 3). The resected material was then sent for histopathology and culture for all patients.

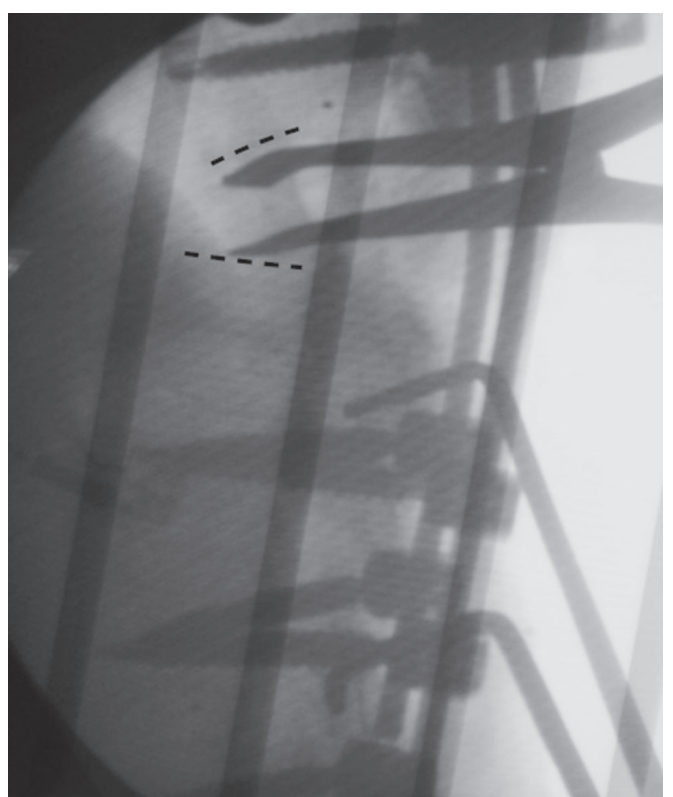

Fig. 4. Fluoroscopic image showing an interbody spreader located between two vertebral bodies following excision of the anterior wedge. The dotted lines represent the lower border of the proximal vertebra and the upper border of the vertebra distal to the excised internal gibbus.

\section{Deformity correction}

\section{1) Stage 1}

The hinge in the operating table was utilized, and the proximal part of the table was extended. The temporary rod can be bent simultaneously using in-situ benders to aid in the correction of the deformity. This leads to SR of the proximal vertebral column.

2) Stage 2: exchanging temporary rods for a final rod The temporary rod on one side was removed, and the contoured final rod was applied on pedicle screws of the distal fragment. The rod was placed in the reduction screws of proximal fragment, and the locking nuts were placed sequentially starting with the most proximal reduction screw. The locking nuts were not fully tightened. The same procedure was repeated on the opposite side. Then, the locking nuts of the reduction screws on both sides were tightened together to bring the proximal segment in alignment with the distal segment. This led to further SR of the proximal vertebral column.

3) Stage 3: controlled anterior opening and posterior closing An interbody spreader was inserted through the lateral costo-transversectomy defect and placed between the inferior surface of the proximal vertebra and the superior 


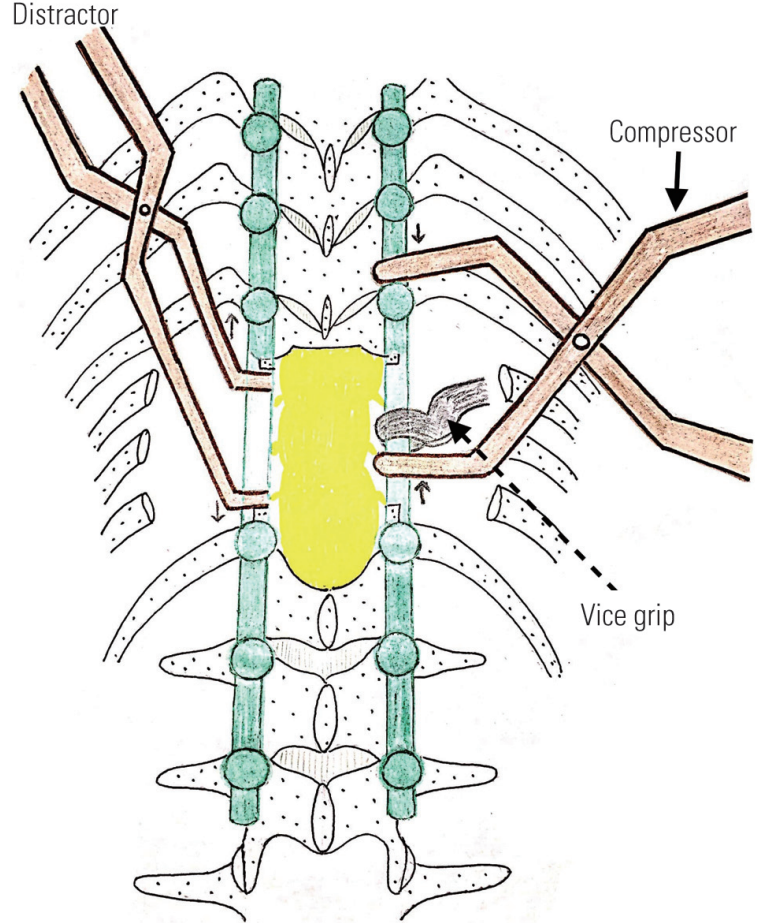

Fig. 5. Schematic diagram showing how the interbody spreader is passed anteriorly through the defect created by costo-transversectomy. If the screws of proximal and distal segments are spaced wide apart, a vice-grip may be applied in the rod and compression may be leveraged against it as shown in the figure.

surface of lower vertebra (Figs. 4-6). A compressor was applied between the distal-most screw of the proximal segment and the proximal-most screw of the distal segment. If the screws are spaced wide apart, a vice-grip may be applied on the rod, and a compressor is applied against the vice-grip (Figs. 5, 6C). The locking nuts of the reduction screws in the proximal fragment were slightly loosened to allow compression-distraction to occur.

Following this, simultaneous AOPC was performed until the end plates of the vertebrae proximal and distal to the excised gibbus became parallel to each other (Fig. 6C). While doing this step, constant vigilance was kept on the anterior dura, which must not be stretched. The use of an expandable anterior cage together with applying posterior compression can make the use of interbody spreaders unnecessary, since the cage can be expanded anteriorly. However, we noticed that the degree of correction was substantially better if an interbody spreader was used for anterior distraction.

Before proceeding with reconstruction, Stagnara's wakeup test was performed to ensure that there was no damage to the spinal cord. Somatosensory evoked potentials

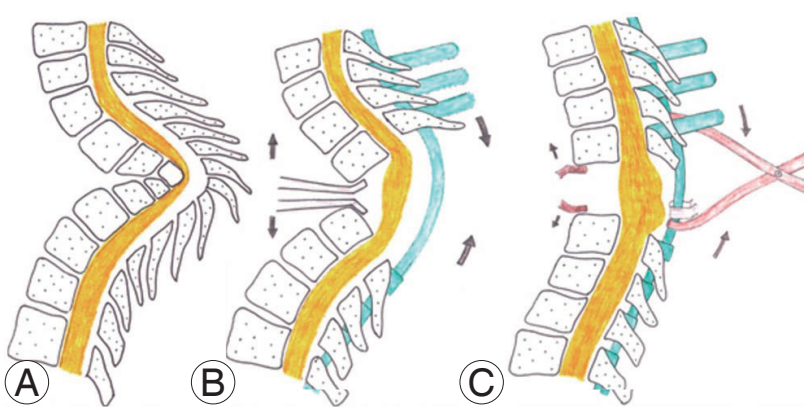

Fig. 6. Schematic diagrams showing steps in VCR with controlled anterior opening and posterior closing maneuver. (A) Status of the vertebral column before VCR. The anterior column in short and posterior column has been lengthened. (B) Status of the vertebral column after VCR. Posterior fixation performed with reduction screws in proximal segment and polyaxial pedicular screws in the distal segment. An interbody spreader is placed anteriorly. The direction of anterior distraction and posterior compression is shown. For schematic representation, the interbody spreader is shown anteriorly, but one must remember that it is inserted from postero-lateral window as shown in Fig. 5. (C) The desired end point of anterior opening posterior closing maneuver, where the end plates of the vertebra proximal and distal to the excised internal gibbus become nearly parallel to each other. If the screws of proximal and distal segments are spaced wide apart, a vice-grip may be applied in the rod and compression may be leveraged against it as shown in the figure. For schematic representation, the interbody spreader is shown anteriorly, but one must remember that it is inserted from postero-lateral window as shown in Fig. 5. VCR, vertebral column resection.

(SSEP) and motor evoked potentials (MEP) are ideal at this stage, but due to unavailability of these modalities at Seth GSMC and KEM Hospital, we performed a wake-up test.

There are no guidelines for the surgeon to know how much to safely stretch the ventral spinal cord given that this deformity is chronic and progressive. SSEP and MEP may be a useful tool to guide the extent of the anterior opening. Since we did not have this facility in our institute, we used manual palpation of the anterior dura using a blunt instrument to guide us while performing the AOPC maneuver. While performing the decompression from 8 o'clock to 4 o'clock, we monitored the tension of the anterior dura over the internal gibbus by palpation with a curved ball tip probe or penfield. After totally removing the internal gibbus, we repalpated the relatively relaxed dura. The aim of gentle anterior distraction is to prevent crowding and kinking of neural structures in its own substance. The posterior compression over a gently distracted anterior column does not allow for neural tissue to become stretched. After posterior compression, when we repalpated the anterior dura for the third time, 
one might feel that there is further softening and laxity on palpation with penfield. Very enthusiastic, uncontrolled anterior distraction can lead to neural stretching and must be avoided. This is a subjective feeling, but for the same operating surgeon, the feel of the anterior dura at three different occasions would definitely help as a reference in guiding the maneuver. To add to this, during per abdominal examination, feeling of the abdominal wall with the pulp of finger is akin to what the normal dura feels like, while a stretched dura feels like a tight tendo-achilles when the foot is plantar flexed.

\section{Anterior reconstruction}

Anterior reconstruction may be done using an autologous strut bone graft or a cage. The maximum limit of the anterior opening is when both end plates proximal and distal to the excised gibbus become parallel. This prevents over distraction of the dural sac. The size of the anterior interbody graft or cage is calculated intraoperatively after completion of the AOPC maneuver. Cortico-cancellous chips obtained during decompression were used to prepare an anterior bed, over which a cage filled with bone graft was placed. The remaining void was filled with cortico-cancellous chips and a layer of gel-foam was placed between the cord and bone graft to prevent the bone graft chips from injuring the spinal cord. If the bone graft is sparse, autologous bone may be harvested from posterior iliac crest.

\section{Posterior reconstruction}

Posterior fusion was performed out at all levels utilized in fixation. This was done using the remaining bone graft

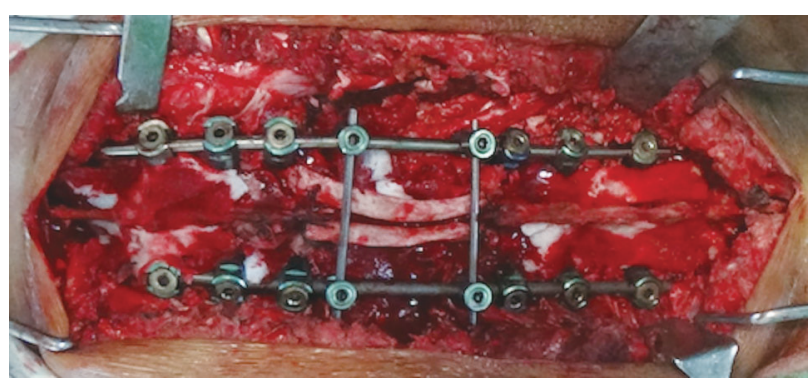

Fig. 7. Intraoperative image showing final fixation and posterior reconstruction. The deformity has now been corrected. Two ribs have been used to bridge the defect caused by posterior decompression. A transverse connector placed over the rib prevents posterior displacement of the rib graft. and a match-stick graft prepared from the excised ribs.

The laminectomy defect was bridged using two ribs (Fig. 7). To prevent migration of the ribs, they were sutured to the adjoining spinous process using bone tunnels.

Following posterior reconstruction, two transverse connectors were applied on the rods. One was applied proximal and the other distal to the site of VCR.

\section{Postoperative management}

Oral feeding commenced once bowel sounds reappeared. Patients were gradually mobilized after applying a total contact brace. The superior extent of the brace was up to the cervicodorsal junction, and distally the brace was molded around the iliac crest on both sides. Ambulation was initiated 3-5 days after surgery in patients having at least grade 4 power in their lower limbs. In patients with non-ambulatory power, bedside sitting and in-bed exercises were started. Sutures were removed on day 14 postoperatively, and all patients were kept in the hospital until suture removal. Two patients who developed superficial maceration of the operative site required an extended stay of 21 days in total.

The brace was discontinued after radiological demonstration of satisfactory anterior union. The clinical and radiological outcomes following surgery in case no. 7 is shown in Figs. 1 and 8.
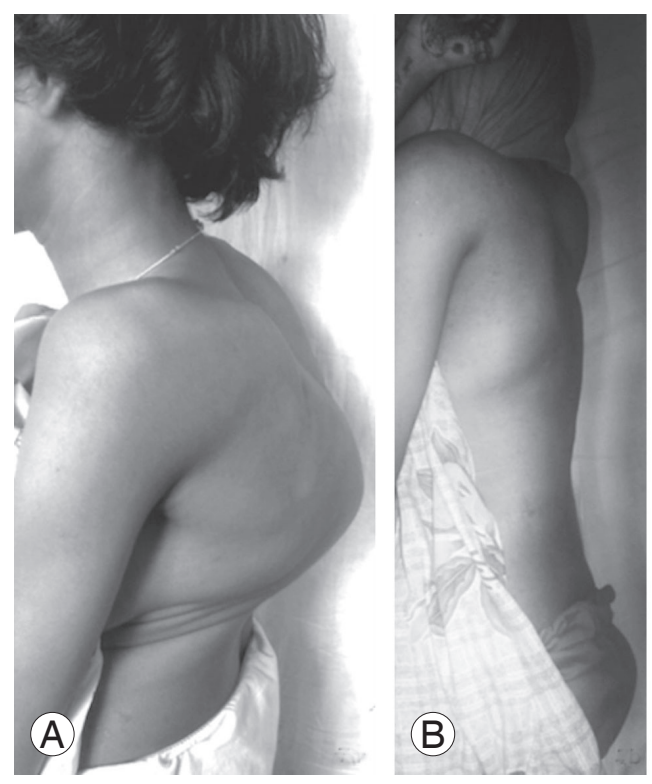

Fig. 8. Clinical images of patient number 7 before (A) and after correction $(\mathbf{B})$ of the deformity showing complete correction of severe kyphosis. 


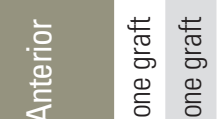 \\ 䓠

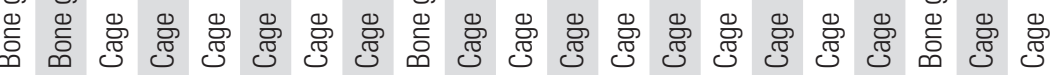

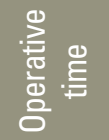

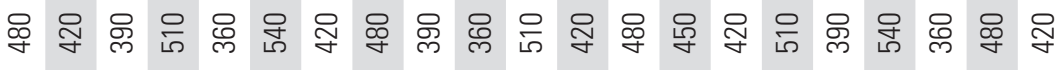 \\ 붐

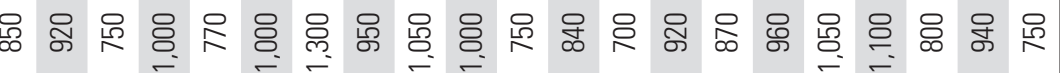

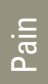 \\ 간 $\sim \sim \sim-\sim--\sim--\sim--\sim \sim-$

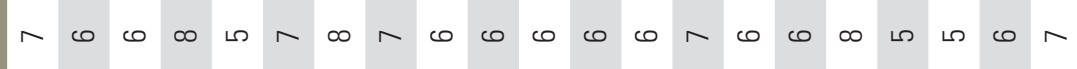 \\ 造

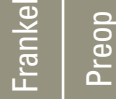 \\ «

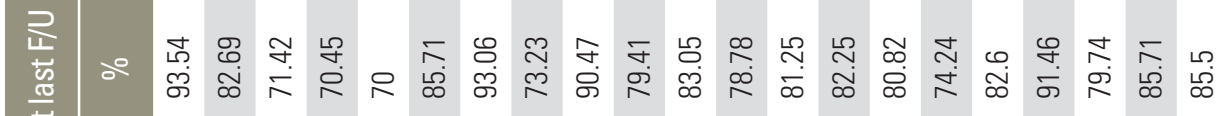

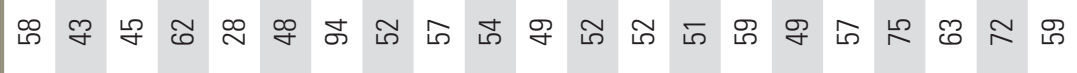

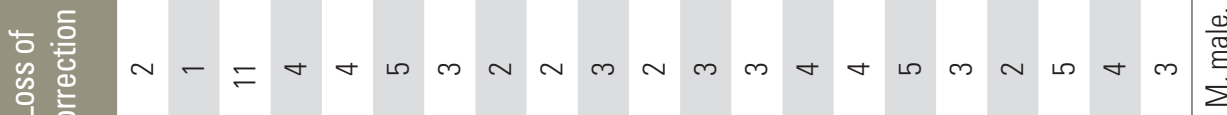

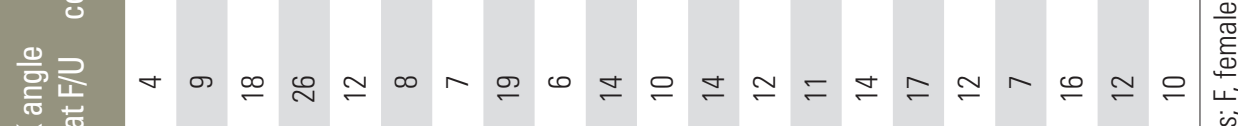

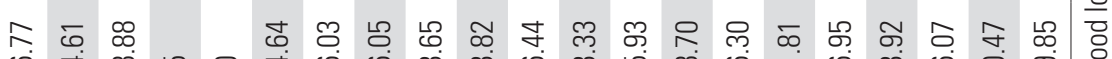
৫ं ळ

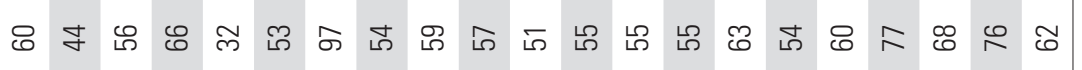




\section{Statistical analysis}

Descriptive statistics including mean and standard deviations were calculated for variables using SPSS ver. 17.0 (SPSS Inc., Chicago, IL, USA). Preoperative K angle and VAS score were compared to postoperative values using Student paired $t$-test.

\section{Results}

This study included 21 patients (eight males and 13 females) with a mean age of $21.90 \pm 7.85$ years (range, 12-43 years). All patients had a minimum follow-up of 18 months. Average follow-up was 30.4 months (range, 18-96 months). No patient had osteoporosis (the T score of all patients was above -2.5 on the DEXA scan). Clinical and radiological data of patients is summarized in Table 1.

All patients had a history of childhood TBS and were treated with chemotherapy and mobilization with brace. The mean duration of chemotherapy was $9.3 \pm 3.12$ months (range, 3-18 months). The resected material in one patient showed histopathological evidence of tuberculosis and subsequent culture showed multi-drug resistant tuberculosis. The patient was successfully treated with combination chemotherapy for 2 years per our institutional protocol. There was no clinical and radiological evidence of recurrent tuberculosis in any patient. The average number of vertebral bodies destroyed was $2.57 \pm 0.51$ (range, 2-3).

The estimated blood loss was $917 \pm 144 \mathrm{~mL}$ (range, $700-1,300 \mathrm{~mL}$ ) and average operative time was $444 \pm 59$ minutes (range, 36-540 minutes). Anterior reconstruction was performed using tricortical iliac crest bone graft in four patients and cage in 17 patients. At the presentation to our clinic, mean preoperative kyphosis angle was $68.42^{\circ} \pm 13.23^{\circ}$ (range, $40^{\circ}-101^{\circ}$ ). Immediately postoperative mean $\mathrm{K}$ angle was $8.71^{\circ} \pm 4.58^{\circ}$ (range, $2^{\circ}-22^{\circ}$ ). Improvement in $\mathrm{K}$ angle postoperatively was statistically significant $(p<0.001)$. The average percentage of correction was $87.11 \% \pm 6.02 \%$ (range, $75 \%-96 \%$ ). We achieved a mean correction of $59.71^{\circ} \pm 12.98^{\circ}\left(\right.$ range, $\left.32^{\circ}-97^{\circ}\right)$. There was no significant difference in improvement of $\mathrm{K}$ angle depending on the number of vertebral bodies destroyed $(p=0.16)$.

At the latest follow-up visit, the mean $\mathrm{K}$ angle was $12.28^{\circ} \pm 5.08^{\circ}$ (range, $4^{\circ}-26^{\circ}$ ) with an average loss of correction of $3.57^{\circ} \pm 2.03^{\circ}$ (range, $1^{\circ}-11^{\circ}$ ). The average correc- tion at the latest follow-up visit was $81.68 \% \pm 7.09 \%$ (range, $70 \%-94 \%)$. Part of this apparent loss in correction could be attributed to the fact that immediate postoperative $\mathrm{X}$ rays were taken in the supine position while the $\mathrm{X}$-rays at follow-up were taken in the standing position.

Thirteen patients did not have any preoperative neurological deficit, which did not change postoperatively. Out of five patients with Frankel grade C, four recovered completely (grade E) but one patient remained as grade $\mathrm{C}$. The average time for recovery in these four patients was 17 weeks (range, 8-36 weeks). Out of the three patients with Frankel grade $\mathrm{A}$, one patient recovered to grade $\mathrm{D}$ and was independently ambulatory with the aid of a walker in 6 months. The other two patients recovered to grade $\mathrm{C}$ (in 4 and 6 months, respectively). No patient had a deteriorated status following surgery.

Pain as judged by VAS score significantly improved from an average preoperative score of $6.38 \pm 0.92$ (range, $5-8$ ) to $1.38 \pm 0.49$ (range, $1-2$ ) at the final follow-up visit $(p<0.01)$. There was no significant difference in improvement of VAS score depending on number of vertebral bodies destroyed ( $p=0.16$ ).

Successful fusion was achieved in all cases. This was confirmed radiologically by the presence of bridging bone between the proximal and distal extent of the VCR (Fig. $1 \mathrm{~B}, \mathrm{C})$.

Two patients experienced superficial wound maceration, which healed with daily cleaning and dressing. One patient developed persistent hypotension post operatively. He was managed in the intensive care unit with intravenous fluids, whole blood, and temporary inotropes along with strict monitoring. The patient eventually made an uneventful recovery. One patient developed a hemothorax postoperatively and was managed by chest drainage tube insertion and regular monitoring. The patient recovered without the need for any additional surgery. As a result of nerve root ligation, patients experienced hypoesthesia in the dermatomal distribution if the ligated nerve root. However, none of the patients complained of radicular pain. The patients regained near complete sensation 6-12 months following surgery, likely due to the overlapping of adjacent dermatomes.

\section{Discussion}

Before the development of the halo-pelvic apparatus, treatment of severe PTK was difficult and dangerous. In 
1974, Yau et al. [8] described the correction of PTK with spinal osteotomy, halo-pelvic distraction, followed by anterior and posterior fusion. The process was long and cumbersome and the average correction achieved was only $28.3 \%$ with serious complications including death of patients. However, with the advancement in surgical techniques and development of pedicle screws, new frontiers have opened up for the correction of PTK.

As described earlier, PTK leads to the shortening of anterior column and lengthening of the posterior column. Only anterior surgery offers advantage of direct under vision anterior debridement; however, achieving correction of severe kyphosis is difficult without strong posterior pedicular screw instrumentation. Moreover, due to old tuberculosis, there may be adhesion within the chest cavity making surgery difficult and is prone to complications. Only posterior osteotomy, like PSO, which shortens the posterior column, is also effective for correction of kyphosis. However, in PTK, due to significant anterior body loss with associated short anterior column, only posterior column shortening will lead to kinking of the cord and increases the risk of neurological deficit.

Osteotomy has the advantage of simultaneously lengthening the anterior column and shortening the posterior column, thus preventing excessive kinking of the spinal cord. One very important aspect of anterior lengthening in parallel to the posterior shortening is that this combination of maneuvers moves the fulcrum from the anterior edge of the vertebral column in a posterior direction. Thus, there is less shortening, less AP translation, and less angular realignment at the level of the spinal cord. This helps to prevent inadvertent spinal cord injury.

There is no doubt that neuromonitoring is the standard of care for severe deformity correction and is vitally important. Since we do not have a neuromonitoring facility in our hospital, we still rely on the age-old wake-up test. The process of instrumentation and VCR is essentially performed by an extremely experienced team of trained anesthesiologists and surgeons. After the temporary rod fixation, over screw VCR is performed diligently with due care. Just prior to the removal of the cortical shell of the internal gibbus, the feeling of the neural tissue on the anterior and anterolateral part of the cord is much like a taut band when it is palpated by a smooth penfield or a ball tip probe. The posterior dura feels soft and relaxed. Once the corrective maneuver is performed the anterior and anterolateral dura appears to be more lax compared
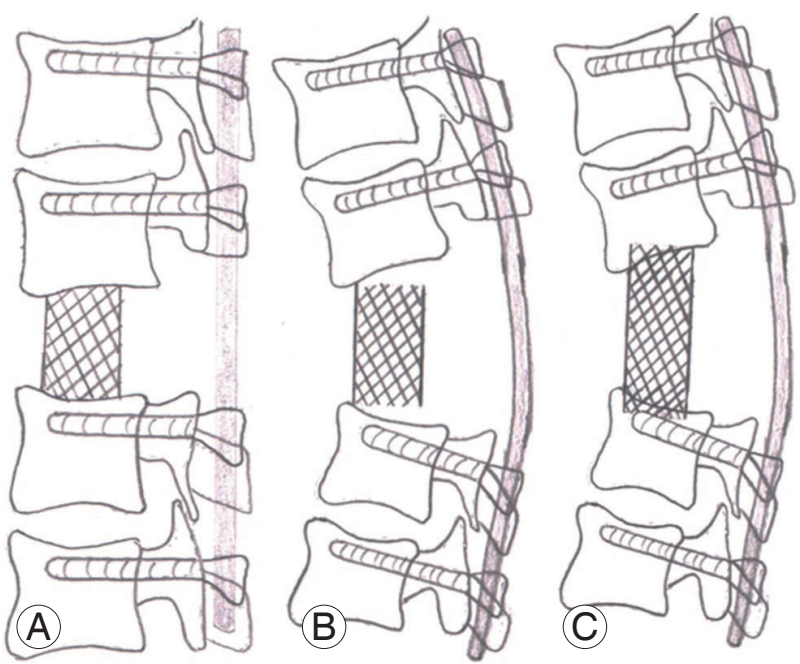

Fig. 9. Schematic diagrams of incomplete and desired correction. (A) Schematic diagram of desired correction with the end plates of vertebra proximal and distal to the excised gibbus are parallel. The cage rests completely on the bone. (B) Schematic diagram of incomplete correction where the end plates of the vertebra proximal and distal to the excised gibbus are not parallel. The cage does not have complete contact with bone. (C) In the case of incomplete correction, if excessive posterior compression is done in an attempt to fit the cage snuggly, the cage may sink into the vertebral body.

to the previous pre-correction tautness. This is essentially because the corrective maneuver is a column shortening procedure. Rather, we should avoid the overcrowding of neural tissue, which usually happens during shortening procedures. This is apparent due to the appearance of wrinkles and insinuation. So, the surgeon can get some idea about the amount of stretch the neural tissue might experience during the described protocol to correct for the deformity. Since the wake-up test is performed after the completion of the deformity correction in absence of neuromonitoring, the only possible way to assess the hazardous stretch of the neural tissue is literally in the hands of the surgeon in a resource-poor setting. Anesthetists always maintain a systolic blood pressure of $>90 \mathrm{~mm} \mathrm{Hg}$. Only spinal surgeons with sufficient experience and methodical training are permitted to perform this surgery.

Incomplete correction is defined as a correction of the deformity, in which the apex of the deformity has not been fully corrected. In severe kyphosis, the end plates of the vertebra proximal and distal to the excised wedge may not become parallel. Due to this, the spacer device used for the anterior reconstruction (cage or bone graft) will not have complete contact with the bone (Fig. 9B). If excessive compression is given, part of the cage might 


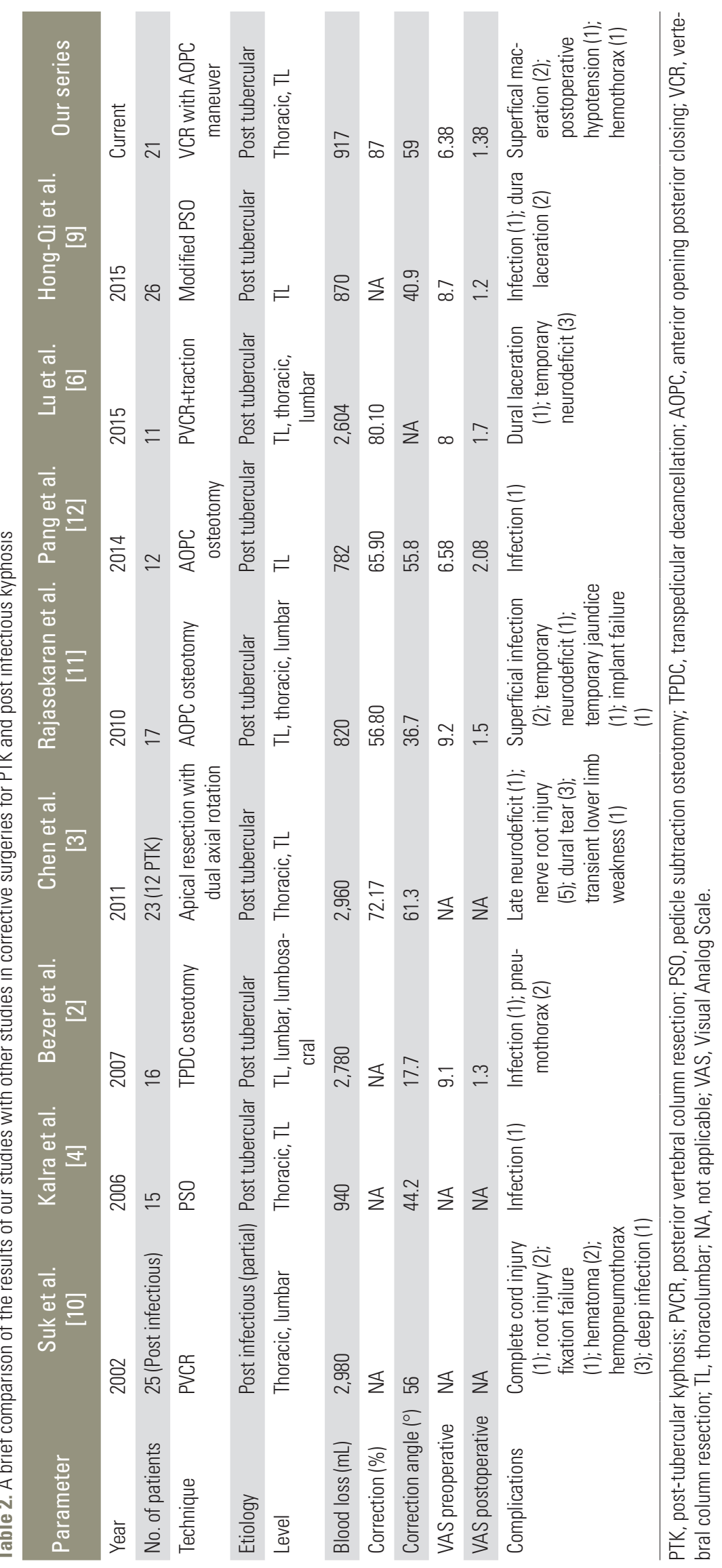


sink into vertebral body (Fig. 9C). Conversely, the desired correction is when the end plates of vertebra proximal and distal to the excised wedge are nearly parallel to each other (Fig. 9A). In cases of desired correction, the anterior spacer will have complete contact with the bone, thus improving the stability of the correction, and also allowing better surface area for vertebral union.

Bezer et al. [2] described transpedicular decancellation osteotomy for PTK and achieved a mean kyphosis correction of $17.1^{\circ}$. Kalra et al. [4] achieved a mean correction of $44.2^{\circ}$ using PSO in PTK. Hong-Qi et al. [9] described a modified PSO and obtained a mean correction of $40.6^{\circ}$. Suk et al. [10] described their of posterior vertebral column resection (PVCR) used for correction in PTK, and although they were able to achieve a mean correction of $56^{\circ}$, mean blood loss was $2,980 \mathrm{~mL}$ and complications were reported in 10 out of their 25 patients. Lu et al. [6] described a technique of PVCR combined with manual intraoperative traction for correction of PTK. Two unscrubbed assistants applied longitudinal traction by pulling on the patient's shoulders and ankles under the drapes provided manual traction. Although they achieved a good radiological correction (a mean correction of $80.1 \%$ ), the average blood loss in their series was 2,604 mL, compared to 914 $\mathrm{mL}$ in our series and $820 \mathrm{~mL}$ and $782 \mathrm{~mL}$ in two other series of AOPC osteotomy. In our opinion, the control of manual traction used to correct deformity is difficult intraoperatively; inadvertent over distraction is possible, which can cause serious damage to spinal cord. We achieved a mean correction of $59.71^{\circ}(87.10 \%)$ and encountered only two major complications, none of which required a secondary revision surgery. Improvement in pain as judged by the VAS score was similar to the series of cases discussed above. A brief comparison of results of our series in the context of the above series is shown in Table 2.

Kawahara et al. [5] was the first to describe AOPC osteotomy for non-infectious kyphosis in 2001. Rajasekaran et al. [11] first described a first series of AOPC osteotomies for PTK in 2010, achieving an average correction of $56.8 \% \pm 14.6 \%$. In 2014, Pang et al. [12] reported a series of 12 patients using same procedure and achieved a mean correction of $65.9 \% \pm 6.35 \%$ compared to the $87.1 \% \pm 6.02 \%$ in our series. We feel that this difference is primarily due to a difference in our respective techniques for anterior distraction. After excision of the wedge, Rajasekaran [7] and Pang et al. [12] used gradual posterior compression to cause simultaneous shortening of the posterior column and opening of the anterior column. In addition to posterior compression, we also used an interbody spreader anteriorly to further open the anterior column, thus achieving greater kyphosis correction. Chen et al. [3] described a similar procedure in 2011 , demonstrating $72.17 \%$ correction in patients with severe angular kyphosis.

The surgical technique described in this paper can potentially be applied to any sharp angular kyphosis. The presence of osteoporosis may be considered a relative contraindication to this procedure as this surgery relies heavily on the good hold of the pedicle screws. Patients with osteoporosis may be treated appropriately prior to considering a deformity correction. Since none of our patients were above 50 years and majority of our patients (12 of 14) were less than 30 years old, we did not face this problem.

We must state that although this procedure is potentially less damaging to the spinal cord, serious complications may arise from the implementation of this procedure if performed by an inexperienced operator. Although we did not experience any major neurological complications, we would like to remind the readers that such corrective osteotomies have potential to cause serious neurological complications, including, but not limited to, irreversible paraplegia. We fully endorse the Scolio-1 Risk Trial and work by Lenke et al. [13] and always counsel the patient and their family regarding the substantial risk of neurological deficit, particularly as these deficits may not be just minor but could include paraplegia. Apart from counseling the patient and family about possible risks, we also actively involve them in vigorous preoperative chest physiotherapy and nutritional care, as most of these patients hail from poor socio-economic strata.

One limitation of our study is the small sample size. Also, due to absence of a control group, direct comparison with other procedures like PSO, modified PSO, or PVCR was not performed. Future prospective comparative studies may provide further insight into the advantages and potential fallacies of these procedures.

\section{Conclusions}

Simultaneous controlled anterior column lengthening and posterior column shortening by an AOPC osteotomy is an effective method for surgical correction of severe PTK. This procedure can be safely performed in a single stage and has been shown to have good clinical and radiological 
outcomes.

\section{Conflict of Interest}

No potential conflict of interest relevant to this article was reported.

\section{References}

1. Benli IT, Acaroglu E, Akalin S, Kis M, Duman E, Un A. Anterior radical debridement and anterior instrumentation in tuberculosis spondylitis. Eur Spine J 2003;12:224-34.

2. Bezer M, Kucukdurmaz F, Guven O. Transpedicular decancellation osteotomy in the treatment of posttuberculous kyphosis. J Spinal Disord Tech 2007;20:209-15.

3. Chen Z, Zeng Y, Li W, Guo Z, Qi Q, Sun C. Apical segmental resection osteotomy with dual axial rotation corrective technique for severe focal kyphosis of the thoracolumbar spine. J Neurosurg Spine 2011;14:106-13.

4. Kalra KP, Dhar SB, Shetty G, Dhariwal Q. Pedicle subtraction osteotomy for rigid post-tuberculous kyphosis. J Bone Joint Surg Br 2006;88:925-7.

5. Kawahara N, Tomita K, Baba H, Kobayashi T, Fujita T, Murakami H. Closing-opening wedge osteotomy to correct angular kyphotic deformity by a single posterior approach. Spine (Phila Pa 1976) 2001;26:391402.

6. Lu G, Wang B, Li Y, Li L, Zhang H, Cheng I. Posterior vertebral column resection and intraoperative manual traction to correct severe post-tubercular rigid spinal deformities incurred during childhood: minimum 2-year follow-up. Eur Spine J 2015;24:586-
93.

7. Rajasekaran S. The natural history of post-tubercular kyphosis in children: radiological signs which predict late increase in deformity. J Bone Joint Surg $\mathrm{Br}$ 2001;83:954-62.

8. Yau AC, Hsu LC, O’Brien JP, Hodgson AR. Tuberculous kyphosis: correction with spinal osteotomy, halo-pelvic distraction, and anterior and posterior fusion. J Bone Joint Surg Am 1974;56:1419-34.

9. Hong-Qi Z, Yong C, Jia H, Chaofeng G, Xiongke H. Modified pedicle subtraction osteotomies (mPSO) for thoracolumbar post-tubercular kyphosis in pediatric patients: retrospective clinical cases and review of the literature. Childs Nerv Syst 2015;31:1347-54.

10. Suk SI, Kim JH, Kim WJ, Lee SM, Chung ER, Nah $\mathrm{KH}$. Posterior vertebral column resection for severe spinal deformities. Spine (Phila Pa 1976) 2002;27:2374-82.

11. Rajasekaran S, Vijay K, Shetty AP. Single-stage closing-opening wedge osteotomy of spine to correct severe post-tubercular kyphotic deformities of the spine: a 3-year follow-up of 17 patients. Eur Spine J 2010;19:583-92.

12. Pang $X$, Li D, Wang $X$, et al. Thoracolumbar spinal tuberculosis in children with severe post-tubercular kyphotic deformities treated by single-stage closingopening wedge osteotomy: preliminary report a 4-year follow-up of 12 patients. Childs Nerv Syst 2014;30:903-9.

13. Lenke LG, Fehlings MG, Shaffrey CI, et al. Neurologic outcomes of complex adult spinal deformity surgery: results of the prospective, multicenter ScoliRISK-1 study. Spine (Phila Pa 1976) 2016;41:204-12. 